\title{
La gestion des savoir-faire de métier chez les pompiers volontaires du Québec
}

\author{
Jacinthe Douesnard $^{\mathrm{a}-\mathrm{e}}$, Laetitia Larouche ${ }^{\mathrm{b}-\mathrm{e}}$, Denis Ouedraogo ${ }^{\mathrm{c}}$, Caroline Gagné ${ }^{\mathrm{d}-\mathrm{e}}$
}

RÉSUMÉ. Cet article présente des résultats et une réflexion préliminaire concernant la dynamique de la gestion des connaissances du métier de pompier volontaire. Ces travailleurs ont peu de moments communs, dans le cadre de leur travail, pour acquérir et échanger des connaissances, tout en évoluant dans un métier où les erreurs sont lourdes de conséquences. De fait, afin de se protéger des risques autant physiques que psychologiques, ces travailleurs doivent maitriser plusieurs connaissances techniques et expérientielles. Comment parviennent-ils à élaborer et à transmettre les savoir-faire de métier nécessaires? C’est au moyen d'entrevues de groupes non directives et d'une analyse herméneutique du discours des pompiers volontaires que cette étude expose certaines limites relatives à la gestion des connaissances dans ce milieu. Le présent article fait état de constats préliminaires entourant la gestion des connaissances dans ce milieu pour ensuite proposer quelques pistes d'amélioration.

ABSTRACT. This article introduces preliminary results and reflection on the dynamics of trade know-bow management of part-time firefighters. These workers barely have the time to acquire and share knowledge, while evolving in a trade where errors are drastically consequential. In fact, in order to protect themselves from both physical and psychological risks, these firefighters must master technical and experiential knowledge. How can they manage to develop them and communicate the necessary trade? It is through non-directive group interviens and an hermeneutic analysis of the speech of part-time firefighters that this study exposes some limitations relating to the knowledge management within this environment. This article reports preliminary findings about knowledge management within this workplace and then suggests ways of improvements.

\section{Problématique}

Certains citoyens résidant en milieu rural portent le titre de pompier, en plus d'assumer les responsabilités qui incombent à leur métier principal. Ces pompiers dits volontaires (volunteer firefighters) représentent près de $80 \%$ de l'effectif total des pompiers du Québec (MSPQ, 2017). Entre leurs mains repose le succès de nombreuses interventions pouvant avoir des répercussions graves sur la vie des gens qu'ils protègent, mais également sur la vie de leurs collègues ou leur propre vie. Or, si la plupart des pompiers volontaires sont passionnés par leur travail (Douesnard, 2012), plusieurs renoncent rapidement à leurs fonctions. Conséquemment, on constate une problématique de rétention de l'effectif chez ces travailleurs dans plusieurs municipalités québécoises (MSPQ, 2012). Outre les conditions de travail éprouvantes et exigeantes, autant sur le plan physique (CCHST, 2017; CNESST, 2017; Daniels et al., 2014; LeMasters et al., 2006), que sur le plan psychologique (BIT, 2018; Kirby, Shakespeare-Finch et Gavan, 2011; Carpenter et al., 2015; Hurrell et al., 2013; Katsavouni et al., 2016; Kehl et al., 2014), ce désenchantement peut être en partie attribuable à un phénomène de perte de confiance en leurs moyens d'affronter la nature imprévisible et dangereuse de plusieurs de leurs interventions (Douesnard et Larouche, 2018). En effet, considérant la nature occasionnelle de leurs interventions, pratiques et formations, l'élaboration et la transmission

\footnotetext{
a Professeure, Ph. D., psychologue organisationnelle, Université du Québec à Chicoutimi

${ }^{\mathrm{b}}$ Doctorante en psychologie organisationnelle, Université de Sherbrooke

c Doctorant en management de projets, Université du Québec à Chicoutimi

d Professeure, Ph. D., Université du Québec à Chicoutimi

${ }^{\mathrm{e}}$ Laboratoire de recherche et d'intervention sur les incidents critiques en milieu de travail (ICMT)
} 
des connaissances revêtent pour ces travailleurs un aspect complexe. Cet article propose d'aborder la dynamique de la gestion des savoir-faire du métier de pompier volontaire en présentant des résultats préliminaires accompagnés d'une réflexion. Ces résultats sont issus d'une recherche de plus grande ampleur menée auprès de cette population et financée par le FRQ-SC (Fonds de recherche québécois - Société et culture). Leur analyse exhaustive est présentée dans le chapitre 3 du livre «Enjeux humains et psychosociaux du travail », sous la direction de Douesnard (2018).

\section{Contexte}

\subsection{Les particularités du métier}

Cuvrant habituellement dans les municipalités de moins de 200000 habitants, les pompiers volontaires du Québec se distinguent de leurs collègues à temps plein, bien que leur mandat soit analogue. Par exemple, ils occupent très peu (ou pas du tout) leur caserne entre les alarmes, comparativement aux pompiers à temps plein qui y demeurent de nombreuses heures lors de leur quart de travail (Douesnard, 2012). De plus, les pompiers volontaires n'exercent leur métier de pompier que lors de situations d'urgence, donc sporadiquement dans une année. Lorsqu'ils quittent leur occupation principale pour se diriger vers l'intervention, ils s'y rendent la plupart du temps avec leur véhicule personnel, privés alors de l'occasion que représente le trajet en groupe, dans le camion, pour faire le point sur l'intervention à venir. Finalement, en plus des tâches relatives au métier de pompier, ces derniers endossent parfois le rôle de premier répondant. En tant que premiers répondants, ils doivent prodiguer les premiers soins aux blessés, dans l'attente des ambulanciers.

Malgré ces différences entre les pompiers volontaires et les pompiers permanents, quelques similitudes peuvent toutefois être constatées dans leurs mandats. De fait, ils doivent maîtriser les feux, effectuer des désincarcérations sur les lieux d'accidents de voiture, des sauvetages en espace clos, des sauvetages nautiques et enfin, assurer la sécurité civile en cas de catastrophe naturelle (inondation, glissement de terrain, etc.). Lorsqu'ils interviennent, ils ont peu de marge de manœuvre, ils doivent réagir rapidement et poser des gestes lourds de conséquences. Qui plus est, les situations dans lesquelles ils interviennent peuvent à tout moment changer de gravité et se dérouler d'une façon imprévue.

\subsection{Un large éventail de savoir-faire}

Devant ce travail complexe et changeant, les pompiers volontaires doivent maîtriser un large éventail de savoir-faire. De fait, chaque intervention implique certaines procédures spécifiques et démarches à accomplir selon le déroulement de l'intervention. Toutefois, en plus de posséder des notions techniques reliées à l'utilisation du matériel d'urgence, ces travailleurs doivent être capables de déroger des procédures pour s'ajuster au déroulement de la situation afin d'agir prudemment et adéquatement. En conséquence, le travail à effectuer requiert le développement de savoirs expérientiels, des habiletés sensorielles, voire une mémoire sensorielle (Douesnard, 2012; Douesnard et SaintArnaud, 2011).

Ces connaissances tacites se traduisent sur le terrain par la nécessité d'être attentifs aux changements auditifs, olfactifs, visuels, car ce sont ces signes qui permettent d'analyser correctement le comportement du feu. Ils doivent savoir lire le feu afin d'être en mesure d'ajuster leurs interventions et protocoles en conséquence (Douesnard, 2012; Douesnard et Saint-Arnaud, 2011). Ils doivent connaître les flammes, être capables de les décoder, de comprendre la fumée, de savoir que telle fumée, selon sa couleur, est plus dangereuse qu'une autre, que telle odeur signifie telle situation d'urgence, etc. puisque l'incendie est une chasse dont les principaux outils pour le combattre sont les habiletés sensorielles des pompiers (Douesnard, 2012). Qui plus est, les pompiers, qui sont également premiers répondants, doivent être en mesure de prodiguer les premiers soins aux victimes d'accidents dans toutes sortes de circonstances, ce qui implique également des savoir-faire relationnels, lorsqu'ils doivent intervenir auprès de la famille de la victime ou directement pour calmer l'anxiété d'une victime. S'ajoutent à ces connaissances des savoir-faire qui relèvent de la créativité, de la capacité d'analyse rapide des situations et même de la capacité adaptative du travailleur. Ainsi, considérant l'unicité de la quasi-totalité des interventions des pompiers volontaires et considérant que ceux-ci œuvrent 
constamment dans l'imprévisibilité, les connaissances théoriques s'avèrent loin d'être suffisantes.

\subsection{Des connaissances difficiles à transmettre}

En ce qui concerne les procédures, les normes, les techniques et les gestes enseignés aux pompiers volontaires, ces connaissances collectives sont formalisées et disséminées à l'aide de formations (transfert direct) officielles en caserne. De façon générale, ces connaissances formalisées sont, selon Basque et Pudelko (2010, p. 112), « liées généralement à des pratiques de travail prescrites dans l'organisation [...] sont décrites et emmagasinées dans les archives, les bases de données, les procédures, les plans, les modèles, les algorithmes; bref dans toutes sortes de documents qui représentent les connaissances "déclarées" ou "verbalisées" sous forme écrite $[\ldots]$ ». C'est ainsi qu'elles sont conservées à travers le temps pour ensuite être transmises lors des formations.

Toutefois, savoir «lire un feu» pour y réagir adéquatement, ou encore savoir rassurer une victime avec un ton de voix approprié et au bon moment, nécessite des savoir-faire particuliers. Ces connaissances sont difficilement accessibles, difficiles à verbaliser (Dupuich-Rabasse, 2002; Duymedjian, 2001; Emery et al., 2000) et volatiles (Aries, Le Blanc et Ermine, 2008). Par contre, elles peuvent être extractives et transférables (Dieng-Kuntz, 2001). De fait, ces connaissances tacites sont usuellement le résultat d'apprentissage dans l'action et au contact de collègues expérimentés. Elles ne s'apprennent pas; elles se construisent au moyen d'échanges entre collègues et se vivent dans l'expérience réelle.

À notre connaissance, aucune étude sur le transfert du savoir-faire des pompiers volontaires du Québec n'est disponible. En revanche, pour les pompiers permanents, l'expérience de l'incendie, de l'intervention d'urgence et le partage des expériences avec les pairs permettent de développer ces aptitudes sensorielles pendant les moments d'échange à la caserne, entre les alarmes (Douesnard, 2012; Douesnard et SaintArnaud, 2011). Plus précisément, cette étude réalisée auprès des pompiers à temps plein a mis en évidence que ces connaissances sont acquises sur le terrain et rediscutées dans le collectif de travail, en dehors des interventions. À cet effet, Dejours (2009) affirme que les savoir-faire de métier sont transmis a posteriori de l'expérience et que le travailleur en prend conscience seulement après-coup: ces pratiques demétier ne s'apprennent donc pas par la formation scolaire, mais bien au cours d'échanges avec des collègues qui ont partagé une expérience commune.

Ainsi, pour ces pompiers permanents, les savoirfaire de métier, guidés par les règles de métier qu'élaborent les collectifs de travail, permettent aux travailleurs de composer avec les exigences émotionnelles élevées de leur travail et les soutiennent dans les actions prises au moment de situations mettant en scène des imprévus, la peur ou la souffrance (Douesnard, 2012; Douesnard et SaintArnaud, 2011). C'est donc dire qu'une élaboration et une transmission saine et efficiente de ces savoirs sont à la fois bénéfiques pour l'équilibre psychique des travailleurs (Douesnard, 2012), mais également pour leur confort dans la tâche, le vivre-ensemble et l'efficacité de leurs interventions (Dejours, 2009). En somme, les savoir-faire nécessaires dans ce corps de métier ont une utilité qui dépasse le travail bien fait puisque cet ensemble d'habiletés spécifiques permet aux pompiers de se sentir compétents et en contrôle des opérations.

A contrario, les pompiers à temps partiel ne partagent pas de temps commun d'échange, n'ayant pas la même proximité au travail que leurs confrères à temps plein. Ainsi, considérant la rareté des interventions, la rareté des moments en caserne avec leurs collègues et la quantité importante de savoir-faire expérientiels à assimiler, comment parviennent-ils à élaborer et à se transmettre les savoir-faire de métier nécessaires?

\section{Méthodologie}

\subsection{Déroulement}

Se déroulant selon un devis de recherche qualitatif, cette étude a été réalisée à l'aide d'entretiens de groupe à caractère non directif (Gavard-Perret et Aubert, 2012). En tout, 5 groupes de pompiers volontaires du Québec provenant de 5 services incendie québécois ont participé à l'étude, pour un total de 43 participants. Parmi ceux-ci, trois groupes étaient constitués de travailleurs occupant également les fonctions de premiers répondants. 
Les entrevues de groupe ont permis d'accéder au vécu subjectif des travailleurs et ainsi de mieux saisir la dimension de l'imaginaire collectif qui structure et donne sens aux expériences vécues en groupe (Gius-Desprairies, 2003). Ces travailleurs sont les mieux placés pour expliquer comment ils vivent le rapport au travail, leurs sources de plaisir et de souffrance (Dejours et Abdoucheli, 1990). Les entrevues se sont déroulées dans les locaux des services incendie visés par la recherche, dans différentes régions administratives du Québec. Chaque groupe a participé à deux rencontres d'une durée approximative de deux heures trente chacune. Au cours de la première entrevue, l'émergence d'une expression libre et spontanée des idées par les participants a été favorisée, permettant prendre connaissance du vécu subjectif des travailleurs, d'aborder avec eux le rapport au travail et à leurs collègues, ainsi que la façon dont les savoir-faire de métier et les pratiques de prudence sont élaborés et transmis. La seconde série d'entrevues permettait, quant à elle, d'approfondir et de clarifier certains éléments qui sont ressortis lors des analyses préliminaires afin de s'assurer d'une compréhension fidèle de leur vécu subjectif. Cette stratégie de validation est préconisée en psychodynamique du travail (Dejours, 2008) et appuyée par Miles et Huberman (2003).

\subsection{Analyse des données}

Aux fins de l'analyse, les entrevues de groupe ont été enregistrées puis transcrites textuellement. Le contenu a d'abord subi une première analyse de traitement de données inspirée de L'Écuyer (1990). Des idées significatives couvrant l'essentiel du matériel ont alors été dégagées du discours, puis décortiquées en unités informationnelles qui, à leur tour, ont été regroupées sous forme de thèmes. Dans un second temps, le travail d'analyse a été fait à partir de l'expression du vécu subjectif des travailleurs, que l'on retrouve dans leurs commentaires, leur interprétation des faits et leurs hypothèses sur le rapport vécu au travail. En ce sens, l'analyse porte "sur ce qui peut être reconnu comme "parole", c'est-à-dire ce qui est formation originale, vivante, affectée, engagée, subjective, émanant du groupe de travailleurs » (Dejours, 2008, p. 201). Ce qui, pour le chercheur, étonne, frappe, surprend, rend mal à l'aise et dérange est considéré comme du matériel significatif sur lequel faire porter une réflexion interprétative. Ainsi, pour approfondir la compréhension de ce qui pousse l'individu à l'action, le schème herméneutique, qui consiste à «développer systématiquement une logique verticale de l'au-delà des apparences ou de la surface des choses » (Berthelot, 1990, p. 73), a également servi de balise dans l'interprétation du discours des travailleurs. Ce travail d'analyse a été réalisé sur le matériel dans le but de faire émerger une compréhension de la dynamique que ces travailleurs entretiennent avec leur métier et leurs collègues. Ces analyses ont par la suite été contre-vérifiées dans un espace de mise en débat scientifique avec la participation d'un groupe de travail réunissant des chercheurs psychodynamiciens du travail. Ce procédé a permis de favoriser l'interprétation intersubjective (Dejours, 2008) et ainsi raffiner notre analyse. Cette analyse du vécu subjectif des travailleurs a permis d'accéder à leur perception de leurs compétences et connaissances et à leur compréhension de la façon dont ils ont développé ces outils.

\section{Résultats préliminaires et réflexions}

Ces résultats préliminaires présenteront les perceptions dichotomiques des groupes de pompiers en regard des processus formels de transmission des connaissances pour ensuite faire le point sur certaines stratégies pouvant favoriser la transmission des connaissances expérientielles du métier.

\subsection{Des formations pratiques insuffisantes}

La formation du pompier (continue, de qualité, approfondie) est une composante indispensable à la pratique du métier de pompier volontaire. Ceuxci doivent suivre une formation initiale et des formations généralement mensuelles en plus de simulations pour exercer le métier de pompiers volontaires. En fait, selon la Loi sur la sécurité incendie, il existe plusieurs types de formation (Gouvernement du Québec, 2015). Et afin de répondre aux exigences de la loi, les pompiers volontaires doivent compléter au moins 375 heures de formation théorique et pratique (Saint-Denis, 2012). Or, les résultats de la présente étude sont cohérents avec les écrits de Dejours (2008) qui suggèrent que ces méthodes formelles sont moins efficaces pour transmettre les savoir-faire expérientiels de métier. De plus, les résultats tendent à démontrer que toutes les brigades de pompiers 
volontaires ne parviennent pas à assurer la transmission des savoir-faire avec le même succès. Il en découle que les équipes qui avaient le plus de difficulté à transmettre les connaissances expérientielles reconnaissaient davantage l'insuffisance des formations théoriques et pratiques.

À titre d'exemple, certaines équipes présentant des caractéristiques d'un fort collectif de travail, au sens de la psychodynamique du travail (Dejours, 2008), démontraient davantage de capacités dans l'échange d'informations utiles et de construction/transmission des savoir-faire. Ces équipes semblaient d'ailleurs valoriser les procédures à titre de protection et exprimaient un confort à les suivre durant les interventions. En ce sens, un pompier mentionnait : "Je ne sens pas que je risque ma vie quand $j$ 'arrive sur une intervention, on est bien protégé, on a des bonnes techniques de travail [...], il y a des procédures à suivre, des manières de faire ", tout comme un autre expliquait que " [éteindre les incendies] n'est pas un stress pour [eux] quand [ils sont] bien encadrés, qu'[ils ont] des marches à suivre ».

À l'opposé, d'autres équipes semblaient se sentir nettement moins outillées pour faire face au caractère imprévisible des interventions et soulignaient l'insuffisance des savoirs formalisés qui leur avaient été enseignés. Par exemple, un pompier nous disait :

«On essaye de notre mieux, pour faire face à ce décalage-là, à cette réalité-là, qui n'est pas écrite dans une procédure. Mais moi, ce qui me chicote, c'est cette évaluation qu'on fait de notre travail, on la fait comment? Parce que je n'ai plus la procédure pour me dire que j'ai bien fait mon travail, j'ai essayé quelque chose, j’ai essayé d'inventer quelque chose où il n'y avait pas de réponse, je l'ai créée $[. .$.$] »$

Certains vont même jusqu'à suggérer que le succès de leurs interventions peut être associé à la chance : «On a été bien plus chanceux qu'on est bons. [...] on a toujours à apprendre, mais on est chanceux, on a toujours eu la chance, à date, il n'y a rien qui nous a explosé en plein visage ».

Or, considérant l'importante quantité de connaissances expérientielles, de même que la nécessité de ces connaissances pour assurer le succès des interventions des pompiers (Douesnard, 2012) et la difficulté de certaines brigades de pompiers volontaires à assurer la transmission de ces savoirs tacites, les brigades de pompiers volontaires gagneraient à améliorer leur management des connaissances, c'est-à-dire le processus nécessaire à la transformation des compétences humaines en capital incorporé (Jacquet, 2010). Pour ce faire, certaines stratégies issues des brigades parvenant à transmettre plus efficacement leurs savoir-faire sensibles pourraient être transposées dans les services en difficulté. À cet effet, les prochaines sections abordent certaines distinctions entre les équipes avec un fort collectif et celles dont le collectif semblait moins consolidé, notamment en regard des méthodes de débriefing technique utilisées et la disposition des services à encourager les membres de l'équipe à se côtoyer et à développer le soutien social.

\subsection{Au-delà du débriefing technique}

On retrouve dans presque toutes les casernes du Québec une pratique qui a cours systématiquement au retour des interventions : le débriefing de l'intervention. Il s'agit d'une séance de discussion où les travailleurs décortiquent les aspects techniques de l'intervention, représentant alors une occasion de retour d'expérience qui favorise l'échange d'informations utiles et nécessaires aux collaborateurs. Cette pratique, bien qu'opérée de façon quasi généralisée, n’est pas exactement la même pour l'ensemble des casernes. Certaines différences apparaissent dans la façon dont elle est organisée, permettant alors de cerner des différences entre casernes relativement à l'utilité et aux retombées de cette séance quant à la transmission des connaissances.

En effet, au-delà des échanges sur les aspects techniques (qui en est l'objectif premier), certains chefs encouragent les échanges et questionnements libres, sincères et spontanés, en sortant du caractère d'évaluation du travail et de recherche de coupables pour les aspects qui ont moins bien fonctionné durant l'intervention, tout en encourageant la verbalisation des émotions ressenties. Cette attitude d'ouverture permet alors de transformer les ressentis des interventions à potentiel traumatisant (dimension pulsionnelle) en un construit utilitaire (connaissances). Il devient donc exploité comme un espace de gestion des connaissances, un marché où se construisent et 
s'échangent de nouvelles connaissances (techniques et émotionnelles), une plate-forme de renouvellement des savoirs de métier toujours éprouvés et constamment questionnés. Lorsque les chefs adoptent cette position d'ouverture, les débriefings semblent avoir des retombées en ce qui a trait à l'apprentissage à partir de l'expérience, à la bonification des connaissances formelles et à la socialisation des travailleurs.

En effet, le débriefing bien utilisé constitue à la fois un processus de retour d'expérience et de rétroaction pour les travailleurs. Par exemple, lorsque les pompiers reviennent sur certains moments critiques de doutes au sujet de leurs interventions, ils partagent avec l'ensemble de l'équipe leurs apprentissages expérientiels de la situation. L'équipe peut alors à la fois apprendre de l'expérience individuelle de ses membres et délibérer au sujet de la situation pour bonifier leur pratique en prévision des prochaines interventions. Les pompiers soulignaient à cet égard l'importance des débriefings pour réguler l'aspect émotionnel des interventions difficiles : «Je me souviens être arrivé à la caserne, le lendemain d'un incident, incapable de raconter l'histoire, trop d'émotions. Je me demandais ce qui se passait. [...] Je me suis dit: "Ça prend un postmortem, comment ça se fait que je n'y ai pas pensé avant!?! "». Qui plus est, il s'agit pour eux d'une façon de veiller sur les nouveaux pompiers qui n'ont pas encore fait l'expérience d'émotions négatives postintervention: " [...] et je me suis dit : "Si j'ai besoin d'un post-mortem... Imagine-toi les nouveaux, il y avait des p'tits nouveaux aussi sur l'intervention "». À cet effet, Bruner (2010) souligne que c'est par la discussion entre collègues que s'opère une domestication de l'erreur et de la surprise, utile notamment pour les travailleurs qui œuvrent dans des contextes instables et changeants. Et, selon Perrenoud (1999), l'apprentissage de la gestion des imprévus s'effectue à travers l'élaboration et la transmission des savoir-faire d'expériences sensibles.

Les grandes catastrophes peuvent également devenir des cas formatifs qui instruisent sur de nouvelles pratiques formelles. En effet, à l'issue des débriefings suivant un événement d'ampleur (par exemple, où il y aurait eu plusieurs blessés graves, ou décès de citoyens ou de pompiers), les éléments rapportés aux gestionnaires permettent d'élaborer de nouvelles procédures ou d'adapter les anciennes en fonction des nouvelles connaissances acquises au moment de l'intervention. Cette expérience se verra alors transmise à l'ensemble des travailleurs et aux générations subséquentes à travers des procédures mises à jour. Il s'agit alors d'une interdépendance entre la création de connaissances et la survenue d'accidents. Ainsi, les interventions construisent l'expérience et l'expérience devient source de connaissances acquises par l'action durant l'intervention. Selon Déry (2013), cette extirpation de la pratique en un savoir tacite et le fait de la recombiner en tâches à organiser et à coordonner est l'acte fondateur du management moderne. Ainsi, le prescrit est revisité, suivant l'expérience du réel partagée par le collectif. Retirer des savoir-faire de l'intervention pour construire de nouvelles procédures afin d'induire de nouvelles pratiques adaptées constitue une forme d'apprentissage collectif. Il s'agirait d'ailleurs d'un principe clé de l'organisation apprenante (Argyris, 1995).

En outre, le débriefing est également un moment de socialisation qui facilite d'une part la mise en relations des travailleurs entre eux, et d'autre part, des travailleurs avec les informations disponibles. Ce moment d'échange permet, pour ceux qui l'utilisent fréquemment, d'asseoir un collectif de travail, tout en favorisant le partage de connaissances et de susciter l'émergence d'une intelligence collective (Dejours, 2009).

En synthèse, le débriefing est à la fois un levier de transfert direct des apprentissages favorisant la transformation des connaissances élaborées en formations et en nouvelles procédures (Basque et Pudelko, 2010) et un dispositif d'autoréflexion et de coconstruction de signifiance partagée, tel qu'envisagé par les tenants de l'apprentissage expérientiel (Experiential learning) comme Dewey $(1916,1938)$ et Kolb $(1976,1984)$. Pour ces derniers, l'expérience se trouve au cœur de l'apprentissage sur le lieu du travail. Le débriefing permet donc de réfléchir sur l'action et de réfléchir dans l'action, tout en étant un processus transactionnel des émotions et des expériences authentiques au retour d'une intervention chargée de connaissances tacites (Rix-Lièvre et Lièvre, 2012). En ce sens, un débriefing mené avec une attitude d'ouverture contribue à la capitalisation des connaissances tacites dans une organisation permettant 
de favoriser la croissance, la transmission et la conservation des connaissances dans cette organisation (Constant, 2012; Soulier, 2005; DiengKuntz, 2001).

\subsection{Encourager le soutien social professionnel}

Une autre distinction retrouvée dans les brigades avec des collectifs plus consolidés est que leurs gestionnaires encourageaient les pompiers à se rencontrer en dehors des interventions, des formations et des pratiques. Ces équipes s'adonnaient à des activités sociales et certaines casernes avaient même aménagé des espaces de repos et de discussion où les pompiers pouvaient se rencontrer en dehors de leurs rares moments de travail. Le temps passé en caserne se trouve être un espace complémentaire au post-mortem pour réguler certaines émotions associées à l'intervention : «Si moi, je ne suis pas prêt à parler lors du post-mortem, ou que je suis correct pendant le post-mortem, mais que c'est trois semaines ou trois mois après que je revois quelque chose qui me redonne un mal de cœur bizarre, qu'est-ce que je fais avec cette émotion-là si je suis tout seul et que je ne vais pas jaser à la caserne et passer du temps là-bas, pour me remettre un peu de ces émotions-là? »

L'importance du temps partagé en caserne fait ici écho aux propos de Dejours (2008) qui affirme que, bien qu'il soit impossible de forcer la création d'un collectif de travail, on peut à tout le moins, en tant que gestionnaire, ne pas y nuire ou tenter de mettre en place les éléments qui en favorisent l'émergence. Ces moments passés en caserne permettent donc aux travailleurs de discuter des interventions, de partager leurs connaissances acquises par l'expérience et de réguler leurs émotions.

\subsection{Pistes de réflexion : un gisement de connaissances inexploitées}

Au-delà des stratégies employées par les brigades parvenant à mieux transmettre leurs connaissances tacites, d'autres avenues tirées de la littérature pourraient faciliter l'exploitation des savoir-faire expérientiels dans le milieu incendie. L'implication des pompiers volontaires dans le perfectionnement de leurs compétences et l'audit du potentiel de connaissances en représentent deux exemples.

En effet, les interviewés ne semblaient pas disposer de plan personnel en regard de leur développement professionnel. D'ailleurs, les démarches personnelles de formation sont à la charge du volontaire et ne sont pas encouragées de façon significative dans le milieu. De plus, aucun dispositif de reconnaissance n'est prévu à cet effet, ni aucun système de veille, qu'il soit institutionnel ou individuel. Il semble que des gisements de connaissances restent inexploités et inexplorés dans le milieu incendie. En effet, non seulement les techniques évoluent en permanence, mais les nouvelles connaissances tacites émergent, et elles gagnent à être partagées pour être réinvesties, permettant ainsi un perfectionnement quotidien, continu et axé sur l'expérience.

En outre, la réalisation d'un audit du potentiel de connaissances s'avère une priorité dans ce milieu. En effet, favoriser l'échange de connaissances entre pompiers volontaires contribue non seulement à la construction d'une procédure de travail efficace et d'une identité de métier, mais également à faire face aux risques du métier collectivement. De fait, les pompiers volontaires jouissent de peu de temps et d'espace pour partager un collectif de travail. La fragmentation, la rareté et la furtivité de leurs rencontres ne favorisent pas, bien souvent, la construction d'un réel collectif de travail, au sens où le décrit la psychodynamique du travail (Dejours, 2008). Dans ces circonstances, il est nécessaire d'établir une stratégie de gestion des savoir-faire de métier, c'est-à-dire un système collaboratif de méthodes et d'outils de gestion de contenu et de travail. Ce système favoriserait la création, la capitalisation et le partage des savoirs et savoir-faire individuels et collectifs dans le milieu incendie.

\section{Conclusion}

Le métier de pompiers volontaires recèle de nombreuses connaissances dont les pompiers sont dépositaires. Or, l'exploitation de ces connaissances est loin d'être optimale. Par leur pratique, ils contribuent à la création de connaissances relatives au métier, savoirs qui leur confèrent un statut de courtier de connaissances (Fortin, 2006) ou de capteur de connaissances (Lefebvre, Roos et Sardas, 2002). Cette gestion des connaissances peut favoriser leur capacité à endosser et vivre ce rôle de pompier. En effet, ce 
savoir contributeur ou créateur de nouvelles connaissances renforce le sentiment d'appartenance et de compétences relationnelles. L'intervention devient alors un lieu d'émergence et de consolidation des connaissances. Elle est un vecteur d'expérience et permet d'opposer les connaissances théoriques aux dures réalités du terrain. Le terrain devient alors le théâtre d'interactions des données concrètes et sensibles. C'est à partir de ces données qu'il est possible, de façon consciente ou inconsciente, pour les pompiers, de construire des connaissances et de les partager avec leurs coéquipiers. Ainsi, la tenue de débriefings permet aux pompiers de verbaliser des connaissances qui peuvent ensuite être réinvesties dans l'élaboration de nouvelles procédures. Toutefois, afin d'exhumer le plein potentiel de cette pratique et d'améliorer la consolidation et la dissémination des connaissances, cette dernière devrait être revue, approfondie, mais surtout formatée de façon à pouvoir rendre accessible à tous les pompiers volontaires cet espace commun d'échanges et de réflexions sur le travail.

\section{RÉFÉRENCES}

Argyris, C. (1995). Savoir pour agir: surmonter les obstacles à l'apprentissage organisationnel. Paris, France : InterEditions.

Aries, S., Le Blanc, B. et Ermine, J.-L. (2008). MASK : une méthode d'ingénierie des connaissances pour l'analyse et la structuration des connaissances. Dans Ermine, J.L. (dir.), Management et ingénierie des connaissances, modèles et méthodes, (p. 208-243).

Basque, J., et Pudelko, B. (2010). La comodélisation de connaissances par objets typés : une stratégie pour favoriser le transfert d'expertise dans les organisations. Télescope, 16,111-129.

Berthelot, J. M. (1990). L'intelligence du social. Paris, France : Presses Universitaires de France.

Bruner, J. (2010). Pourquoi nous racontons-nous des bistoires ?: le récit, au fondement de la culture et de l'identité. Paris, France : Retz.

Bureau international du travail (2018). Directives sur le travail décent dans les services d'urgence. Département des politiques sectorielles, Genève, 46.

Carpenter, G. S. J., Carpenter, T. P., Kimbrel, N.A., Flynn, E.J., Pennington, M.L., Cammarata, C., ... Gulliver, S.B. (2015). Social support, stress, and suicidal ideation in professional firefighters. American Journal of Health Behavior, 39, $191-196$.

Centre canadien d’hygiène et de sécurité au travail (2017, décembre). Les risques pour la santé et sécurité des pompiers. Repéré à http://www.cchst.ca/oshanswers/occup_workplace/firefighter.html

Commission des normes, de l'équité, de la santé et sécurité au travail (2017, décembre). Risques liés à la lutte aux incendies. Repéré à http://www.csst.qc.ca/prevention/secteur/securite-incendie/Pages/risques-incendies.aspx

Daniels, R. D., Kubale, T. L., Yiin, J. H., Dahm, M. M., Hales, T. R., Baris, D., ... Pinkerton, L. E. (2014). Mortality and cancer incidence in a pooled cohort of US firefighters from San Francisco, Chicago and Philadelphia (1950-2009). Occupational and Environmental Medicine, 71, 388-397.

Dejours, C. (2008). Travail, usure mentale : essai de psychopathologie du travail. Paris, France : Bayard.

Dejours, C. (2009). Travail vivant. Paris, France : Payot.

Dejours, C. et Abdoucheli, É. (1990). Itinéraire théorique en psychopathologie du travail. Prévenir, 20, 127-149.

Déry, R. (2013). Les fondements de la gestion des connaissances. Communication présentée au Congrès de l'Association pour la gestion des connaissances dans la société et les organisations, Montréal, Canada.

Dewey, J. (1916, 1938). Experience and education. New York, NY: MacMillan.

Dieng-Kuntz, R. (2001). Méthodes et outils pour la gestion des connaissances : une approche pluridisciplinaire du knowledge management. Paris, France : Dunod.

Douesnard, J. (2012). Métier à risque, la santé psychologique des pompiers. Québec, Québec : Presses de l'Université du Québec.

Douesnard, J. (2018). Enjeux humains et psychosociaux du travail. Québec, Québec : Presses de l'Université du Québec.

Douesnard, J. et Larouche, L. (2018). Défis et enjeux dans l'élaboration des collectifs de travail des pompiers volontaires du Québec. Dans Douesnard, J. (dir.), Enjeux bumains et psychosociaux du travail (p. 43-74). 
Douesnard, J., et Saint-Arnaud, L. (2011). Le travail des pompiers : un métier au service de l'autre. Travailler, 26, 35-53.

Dupuich-Rabasse, F. (2002). Gestion des compétences et knowledge management. Paris, France : Éditions Liaisons.

Duymedjian, R. (2001, mars). Les règles d'or du knowledge management. L'express l'expansion. Repéré à http://lexpansion.lexpress.fr/actualite-economique/les-regles-d-or-du-knowledge-manager $1394020 . h$ tml

Emery, P., Stanewsky, R., Helfrich-Förster, C., Emery-Le, M., Hall, J. C. et Rosbash, M. (2000). Drosophila CRY is a deep brain circadian photoreceptor. Neuron, 26, 493-504.

Fortin, J. (2006). Modèle régional de la Montérégie dans la coordination des connaissances. Le point en santé et services sociaux, 6, 43-46.

Gavard-Perret, M.-L., et Aubert, B. A. (2012). Méthodologie de la recherche en sciences de gestion : réussir son mémoire ou sa thèse, Montreuil, France : Pearson.

Gius-Desprairies, F. (2003). L'imaginaire collectif. Toulouse, France : Érès.

Gouvernement du Québec. (2015, juin). Règlement sur les conditions pour exercer au sein d'un service de sécurité incendie municipal. Repéré à http://www2.publicationsduquebec.gouv.qc.ca/ dynamicSearch/telecharge.php?type $=3 \&$ file $=/ \mathrm{S} \quad 3 \quad 4 / \mathrm{S} 3 \quad 4 \mathrm{R} 1 . \mathrm{htm}$

Hurrell, J. J., Huynh, J. Y., Xanthopoulou, D., et Winefield, A. H. (2013). Social support moderates the impact of demands on burnout and organizational connectedness: A two-wave study of volunteer firefighters. Journal of Occupational Health Psychology, 18, 9-15.

Jacquet, S. (2010, janvier). Le management des connaissances : des clés pour comprendre. Management. Repéré à http://www.creg.ac-versailles.fr/spip.php?article442

Katsavouni, F., Bebetsos, E., Malliou, P., et Beneka, A. (2016). The relationship between burnout, PTSD symptoms and injuries in firefighters. Occupational Medicine, 66, 32-37.

Kehl, D., Knuth, D., Hulse, L. et Schmidt, S. (2014). Posttraumatic reactions among firefighters after critical incidents: Cross-national data. Journal of Aggression, Maltreatment \& Trauma, 23, 842-853.

Kirby, R., Shakespeare-Finch, J. et Gavan, P. (2011). Adaptive and maladaptive coping strategies predict posttrauma outcomes in ambulance personnel. Traumatology, 17, 25-34.

Kolb, D. A. (1976). The learning style inventory: Technical manual. Boston, MA: McBer \& Co.

Kolb, D. A. (1984). Experiential learning. Englewood Cliffs, NJ: Prentice-Hall.

L'Écuyer, R. (1990). Méthodologie de l'analyse développementale de contenu : méthode GPS et concept de soi. Québec, Québec : Presses de l'Université du Québec.

Lefebvre, P., Roos, P. et Sardas, J.-C. (2002, mai). From the management of expertise to the management of design metier. Communication présentée au EURAM Conference Stockholm.

LeMasters, G. K., Genaidy, A. M., Succop, P., Deddens, J., Sobeih, T., Barriera-Viruet, H., ... Lockey, J. (2006). Cancer risk among firefighters: A review and meta-analysis of 32 studies. Journal of Occupational and Environmental Medicine, 48, 1189-1202.

Miles, B. et Huberman, A. (2003). Analyse des données qualitatives. Bruxelles, Belgique : De Boek Université.

Ministère de la Sécurité publique du Québec (2017, décembre). Bottin des services incendie. Repéré à https://www.securite publique.gouv.qc.ca/securite-incendie/bottin.html

Ministère de la Sécurité publique du Québec (2012). Actes du forum sur la sécurité incendie. Les services de sécurité incendie tournés vers l'avenir. Repéré à https://www.securitepublique.gouv.qc.ca/securite-incendie/quebec/forum-sur-la-securiteincendie/actes.html

Perrenoud, P. (1999). Transférer ou mobiliser ses connaissances? D'une métaphore à l'autre : implications sociologiques et pédagogiques. Repéré à http://www.unige.ch/fapse/SSE/teachers/perrenoud/php main/php 1999/1999 28.html

Rix-Lièvre, G. et Lièvre, P. (2012). La dimension « tacite » des connaissances expérientielles individuelles : une mise en perspective théorique et méthodologique. Management international/International Management/Gestiòn Internacional, 16, 21-28. 
Soulier, E. (2005). Le système de gestion des connaissances pour soutenir le storytelling dans l'entreprise. Revue française de gestion, 6, 247-264.

St-Denis, K. (2012). Portrait sociologique de la profession des pompiers du Québec. Rapport de recherche, Repéré à http://collections.banq.qc.ca/ark:/52327/bs2243145 